\title{
Controllable Terahertz Radiation from a Linear-Dipole-Array Formed by a Two-Color Laser Filament in Air
}

\author{
Zhelin Zhang ${ }^{1,2}$, Yanping Chen ${ }^{1,2},{ }^{*}$ Min Chen ${ }^{1,2}$, Zhen Zhang ${ }^{1,2}$, Jin $\mathrm{Yu}^{1}$, Zhengming Sheng ${ }^{1,2,3}$, and Jie Zhang ${ }^{1,2}$ \\ ${ }^{1}$ Key Laboratory for Laser Plasmas (Ministry of Education), \\ Department of Physics and Astronomy, Shanghai Jiao Tong University, Shanghai 200240, China \\ ${ }^{2}$ Collaborative Innovation Center of IFSA, Shanghai Jiao Tong University, Shanghai 200240, China and \\ ${ }^{3}$ Department of Physics, SUPA, University of Strathclyde, Glasgow G4 ONG, UK
}

(Dated: November 10, 2016)

\begin{abstract}
We have demonstrated the effective control on carrier-envelope phase, angular distribution as well as peak intensity of a nearly single-cycle terahertz pulse emitted from a laser filament formed by two-color, the fundamental and the corresponding second harmonics, femtosecond laser pulses propagating in air. Experimentally, such control has been performed by varying the filament length and the initial phase difference between the two-color laser components. A linear-dipole-array model, including the descriptions of the both generation (via laser field ionization) and propagation of the emitted terahertz pulse, is proposed to present a quantitative interpretation of the observations. Our results contribute to the understanding of terahertz generation in a femtosecond laser filament and suggest a practical way to control the electric field of terahertz pulse for potential applications.
\end{abstract}

Terahertz (THz) radiation is of enormous interest for a variety of promising applications such as remote $\mathrm{THz}$ sensing and imaging, THz spectroscopy, THz nonlinear high field physics, and diagnostic of high power laser-matter interaction [1-5]. High peak-power broadband $\mathrm{THz}$ radiations can be generated by two-color laser-induced air-plasmas which can be moreover delivered to remote targets by laser self-guiding, avoiding thus the strong absorption of $\mathrm{THz}$ radiation by water vapor in the atmosphere [6-9]. THz radiation generation in airplasmas can be mainly described using four-wave rectification [10-12] and transient photocurrent [13-21] models. In particular, it is well known from previous studies that the electric field of a THz pulse emitted from a two-color laser-plasma is usually with a single-cycle waveform. Thus, a precise control of the carrier-envelope phase (CEP) is crucial for applications, e.g., THz nonlinear optics and spectroscopy on various systems [22-24].

In this Letter, we have performed an effective control of the spatio-temporal structure of a $\mathrm{THz}$ pulse emitted from a twocolor laser-induced air-plasma channel (filament), including its electrical field waveform (or CEP) and angular distribution as well as its peak intensity. Such control was realized through a change of the filament length as well as the initial phase difference between the two-color laser components. A theoretical model has been developed to describe both the generation and the propagation of the $\mathrm{THz}$ wave emitted from a filament, where the $\mathrm{THz}$ radiation is considered as a coherent superposition of $\mathrm{THz}$ waves emitted from a linear-dipole-array (LDA) along the filament. A good agreement has been obtained between the experimental observations and the predictions from the theoretical model.

A laser pulse of $40 \mathrm{fs}$ pulse duration and $3.5 \mathrm{~mJ}$ energy at $800 \mathrm{~nm}$ was focused by a plano-convex lens of $50 \mathrm{~cm}$ focal length to form a filament in air. Before the laser pulse undergoing the filamentation $[25,26]$, it was intercepted by a $\beta$-barium borate (BBO) crystal of $0.1 \mathrm{~mm}$ thickness, leading to second harmonic (SH) generation as shown in Fig.1(a).

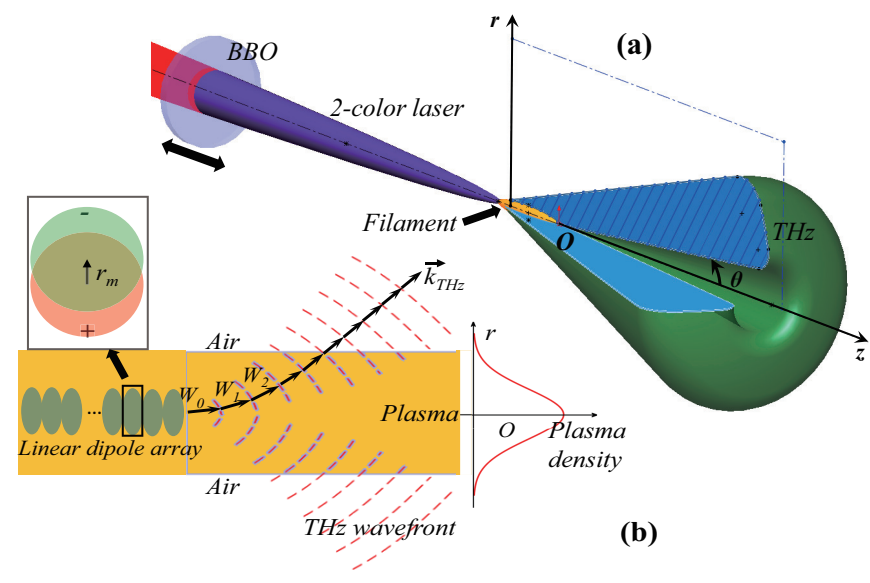

FIG. 1. (a) Schematic presentation of the experimental setup in a polar coordinate $(z, \theta)$ where the origin $O$ corresponds to the geometric focus of the lens. The $z$-axis corresponds to the propagation axes of the laser beam as well as the THz radiation. $\theta$ and $r$ are used to describe the transverse propagation direction of the $\mathrm{THz}$ radiation. (b) Evolution of a THz wavefront (red dashed curves) while a $\mathrm{THz}$ wave passing through different layers of a plasma channel. $W_{0}, W_{1}, W_{2} \ldots$ are typical points on one trace of a $\mathrm{THz}$ wave. The inset shows a dipole formed by charge separation inside a laser-plasma filament.

The initial phase difference $\left(\Delta \phi_{i}\right)$ between the fundamental wave (FW) and its SH could be controlled by translating the BBO along the propagation axis of the laser: $\Delta \phi_{i}=\omega\left(n_{2 \omega}-\right.$ $\left.n_{\omega}\right) d / c$, where $\omega$ is the angular frequency of the FW, $n_{\omega}$ and $n_{2 \omega}$ are the refractive indexes of the FW and its $\mathrm{SH}, c$ the speed of light in vacuum, and $d$ the distance between the BBO and the geometric focus of the lens. The extraordinary axis of the $\mathrm{BBO}$ was oriented at an angle of $50^{\circ}$ with respect to the polarization of the incident FW. The THz emission from the two-color air-filament with polarization parallel to the polarization of the incident FW was detected with an electro-optic sampling technique after being collected by a pair of off-axis parabolic mirrors. 
When the energy of the pump laser is lower than the threshold of the filamentation in air, a short plasma channel is observed around the geometric focus of the focusing lens [Fig.2(a)]. The THz waveform emitted from such plasma channel as a function of $\Delta \phi_{i}$ is illustrated in Fig.2(d). Each vertical line corresponds to a measured $\mathrm{THz}$ waveform at a specified position of the BBO, thus a specific initial phase difference between the FW and its $\mathrm{SH}$ at the geometric focus. The experimental result obtained with the short plasma channel shows that the amplitudes of the $\mathrm{THz}$ waves change as a sinusoidal function of $\Delta \phi_{i}$, i.e., $E_{T H z} \sim \sin \left(\Delta \phi_{i}\right)$, which is consistent with previous reports $[10,13]$. When $\Delta \phi_{i}=0, \pm \pi, \ldots$, the amplitude of the THz wave vanishes. Meanwhile, the CEP of the $\mathrm{THz}$ waveform stays constant when $\Delta \phi_{i}$ changes from $-\pi$ to 0 . For $\Delta \phi_{i}$ changing from 0 to $\pi$, the CEPs of the $\mathrm{THz}$ pulses also keep at a same value with an opposite polarity of the THz fields. When the short plasma channel turns to a long filament by increasing the energy of the pump laser [Fig.2(b,c)], the evolution of the THz waveforms versus $\Delta \phi_{i}$ at the geometric focus becomes more complicated [Fig.2(e,f)]. On one hand, the amplitude of the $\mathrm{THz}$ signal does not obey the sinusoidal relation with $\Delta \phi_{i}$ any more, i.e., its minimum never goes to zero. On the other hand, the CEPs of the $\mathrm{THz}$ waveforms start to change smoothly with $\Delta \phi_{i}$. These observations suggest that efficient control of the CEP of the $\mathrm{THz}$ radiation from a two-color filament can be realized by adjusting the filament length. Stabilization of the CEP of the $\mathrm{THz}$ pulse is easily implemented by making a short plasma channel. In contrast, continuous tunability of the CEP of the $\mathrm{THz}$ pulse can be introduced by manipulating the initial relative phase of the two-color laser components in the case of a long filament.

To understand these new phenomena, a model including the generation and propagation of the $\mathrm{THz}$ radiation from a long filament is investigated. We consider a two-color laser pulse whose electric field parallel to the polarization of the incident $\mathrm{FW}$ is given as $E_{\text {laser }}(t)=E_{\omega} \cos \left(\omega t+\phi_{0}\right)+$ $E_{2 \omega} \cos \left[2\left(\omega t+\phi_{0}\right)+\Delta \phi\right]$, where $E_{\omega}$ and $E_{2 \omega}$ are respectively the amplitudes of the FW and its $\mathrm{SH}, \omega$ and $\phi_{0}$ are respectively the angular frequency and the phase of the $\mathrm{FW}$, $\Delta \phi=\Delta \phi_{i}+\omega\left(n_{2 \omega}-n_{\omega}\right) L / c$ is the relative phase between the two-color laser components while propagating in a plasma filament. Here, $L$ is the propagation distance. As $E_{\text {laser }}(t)$ is used to ionize the gas target, the ionization process is simulated using the ADK model [15, 27]. So we get the density of free electrons born at the time of $t^{\prime}$ at the given longitudinal position $z$ of the filament $d \rho_{e}\left(z, t^{\prime}\right)=w\left(t^{\prime}\right) n_{0}\left(t^{\prime}\right) d t^{\prime}$, where $w\left(t^{\prime}\right)$ is the ionization rate associated with $E_{\text {laser }}\left(t^{\prime}\right)$, and $n_{0}\left(t^{\prime}\right)$ is the density of neutral particles.

For a small fraction of the long filament marked as $d z$, the charge can be written as $d q\left(z, t^{\prime}\right)=-e d \rho_{e}\left(z, t^{\prime}\right) d z$, where $e$ is the elementary charge. After the laser pulse, the free electrons produced from ionization will have a drift velocity given by $v\left(t^{\prime}\right)=\int_{t^{\prime}}^{\infty}-e E_{\text {laser }}(t) / m_{e} d t$, where $v\left(t^{\prime}\right)$ represents the drift velocity for electrons born at the time $t^{\prime}$, and $m_{e}$ is the electron mass. Obviously, the drift motion of free electrons will lead
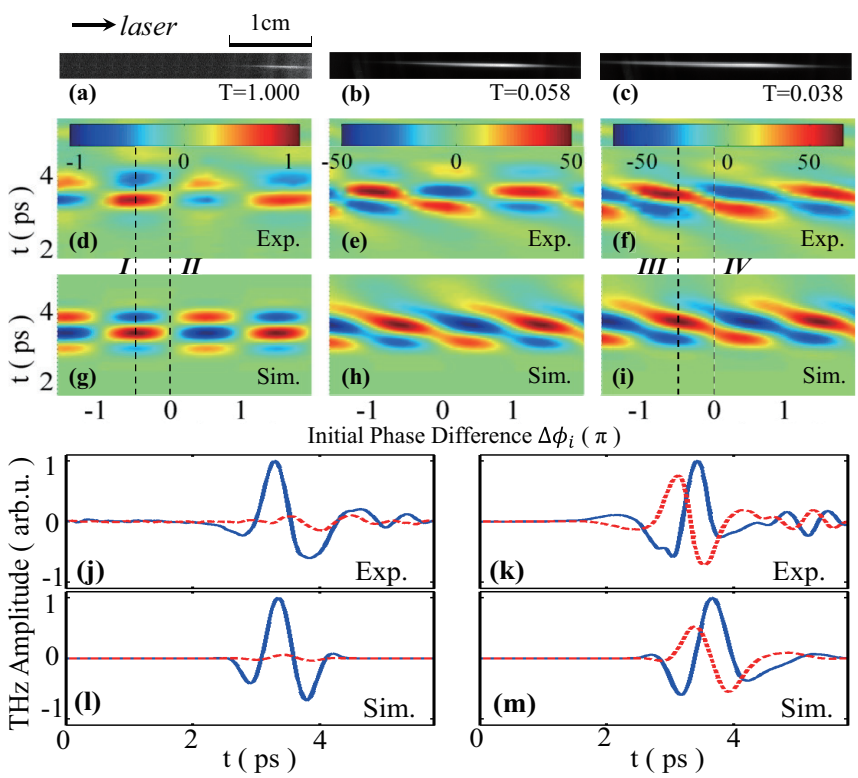

FIG. 2. The pictures of the plasma columns obtained with laser energy of (a) $0.2 \mathrm{~mJ}$, (b) $1.4 \mathrm{~mJ}$, and (c) $2.4 \mathrm{~mJ}$, respectively. $\mathrm{T}$ is the transmittance of the beam before reaching the CCD camera. The length of the plasma column is $3 \mathrm{~mm}$ for (a), $20 \mathrm{~mm}$ for (b), and 30 $\mathrm{mm}$ for (c). The corresponding measured $\mathrm{THz}$ waveforms versus the initial phase difference between the FW and its $\mathrm{SH}$ are given in (d), (e) and (f) while the simulation results are shown in (g), (h) and (i). Typical $\mathrm{THz}$ waveforms obtained when $\Delta \phi_{i}=-\pi / 2$ (solid curve) and $\Delta \phi_{i}=0$ (dashed curve) with (j) a 3-mm-long plasma and (k) a 30-mm-long filament. Corresponding simulation results are shown in (1) for a 3-mm-long plasma and (m) for a 30-mm-long filament.

to the charge separation inside the filament, as schematically illustrated in Fig.1(b,inset). For each single free electron, the final drift distance can be estimated according to the plasma electron oscillations under the restoring force due to charge separation. The quiver amplitude of each electron is simply about $r_{m}=v\left(t^{\prime}\right) / \omega_{p} \sim 1 \mu m$ under the laser intensity around $10^{15} \mathrm{~W} / \mathrm{cm}^{2}$ and the radiation frequency around $1 \mathrm{THz}$, where $\omega_{p}$ is the plasma frequency.

As mentioned above, the separation of the positive and negative charge center with the distance of $\left|r_{m}\right|$ will lead to the formation of dipoles inside the plasma. And the dipole varies as $d P\left(z, t^{\prime}, t\right)=r_{m} d q\left(z, t^{\prime}\right) \exp \left(-j \omega_{T H z} t\right)$, where $\omega_{T H z}$ is close to the plasma oscillation frequency $\omega_{p}$, and $j$ is the imaginary unit. Since $\left|r_{m}\right|$ is typically much smaller than the wavelength of the plasma oscillation with the frequency of several $\mathrm{THz}$, the radiation caused by such oscillation can be considered as a dipole radiation [28]. In polar coordinates, the radiation of a dipole, with oscillation direction perpendicular to the polar axis [z-axis in Fig.1(a)], can be written as

$$
\begin{gathered}
d E_{\text {dipole }}^{T H z}\left(\omega_{T H z}, z, t^{\prime}, t\right)=\frac{1}{4 \pi \varepsilon_{0} c^{3} R(z, \theta)} \\
\times \frac{\partial^{2}}{\partial t^{2}}\left[d P\left(z, t^{\prime}, t\right)\right] \exp [j \Phi(z, \theta)] \cos \theta
\end{gathered}
$$

where $d E_{\text {dipole }}^{T H z}\left(\omega_{T H z}, z, t^{\prime}, t\right)$ represents the $\mathrm{THz}$ electric field 
emitted by the dipole oscillations along the $\theta$ direction which are generated from $d q\left(z, t^{\prime}\right), R(z, \theta)$ is the propagation length of the THz wave from its original position $z, \Phi(z, \theta)$ is the wave phase of the radiation. For a long filament, the whole $\mathrm{THz}$ signal is an integral of the radiation from each dipole located along the filament, i.e., coherent superposition of $\mathrm{THz}$ waves from a linear-dipole-array (LDA). Here we assume that $\mathrm{THz}$ radiation emitted by the filament at the propagation coordinate $z$ does not affect the emission at $z+d z$. Thus,

$$
E_{T H z}\left(\omega_{T H z}, t\right)=\int_{0}^{\tau} \int_{\text {filament }} d E_{\text {dipole }}^{T H z}\left(\omega_{T H z}, z, t^{\prime}, t\right)
$$

where the integration ranges are the pulse duration of the pump and the filament length.

To get the total $\mathrm{THz}$ radiation in far field, not only the generation of the $\mathrm{THz}$ waves from each dipole along the filament but also the propagation and coherent addition of these radiations should be taken into account. The latter can be made by considering the phase propagation term $\exp [j \Phi(z, \theta)]$ in Eq.(1), which is calculated with the ray tracing method as schematically shown in Fig.1(b). As the $\mathrm{THz}$ wave propagates from $W_{0}$ to $W_{1}\left(z+\delta z, r+\delta z \tan \theta_{0}\right)$, its phase changes to $\Phi\left(W_{1}\right)=\Phi\left(W_{0}\right)+k_{0} \eta(z, r) \delta z / \cos \theta_{0}$, where $k_{0}=\omega_{T H z} / c$ is the THz wave number in the vacuum, and $\theta_{0}$ is the radiation angle. The refractive index of the plasma is $\eta(z, r)=$ $\sqrt{1-n_{e} e^{2} / \varepsilon_{0} m_{e} \omega_{T H z}^{2}}$ where $n_{e}$ is the plasma density taking the form of a cosine-squared distribution as $n_{e}(z, r) \sim$ $\cos ^{2}\left(\pi r / 2 r_{p}\right)$ with $r_{p}$ representing the radius of the cross section of a filament. By repeating this process along the trace, one can get the THz wave phase $\Phi(z, \theta)$ in far field. After considering contributions from all dipoles along a LDA by using Eq.(2), we obtain the coherent emission of the $\mathrm{THz}$ waves from the whole filament in far field.
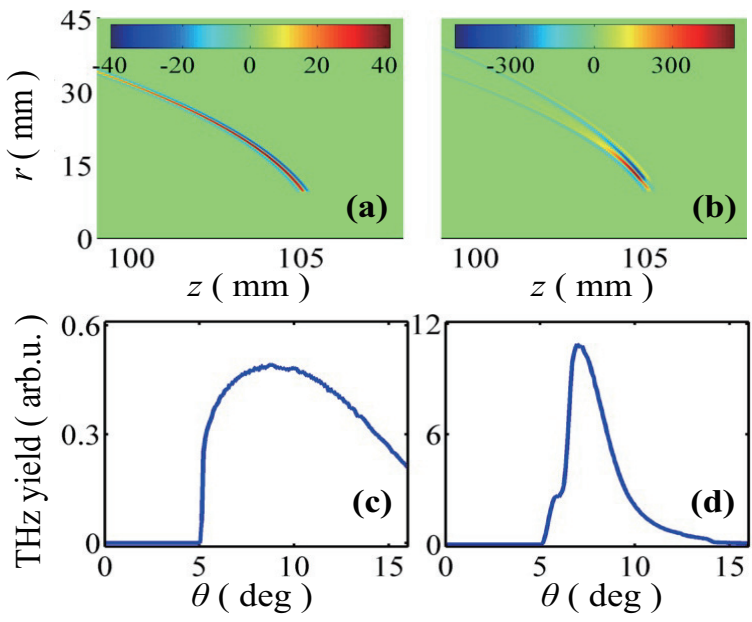

FIG. 3. Spatial distributions of the THz electric field for (a) a 3-mmlong plasma and (b) a 30-mm-long filament. The r-z plane is defined in Fig.1(a). Angular distributions of the intensity of the THz emitted from (c) the 3-mm-long plasma and (d) the 30-mm-long filament, respectively.

Based on this model, simulation results of $\mathrm{THz}$ radiation from a filament versus the initial phase difference $\Delta \phi_{i}$ at the geometric focus for the plasma length of $3 \mathrm{~mm}, 20 \mathrm{~mm}$, and $30 \mathrm{~mm}$ are shown in Figs.2(g,h,i), respectively. The simulation results are coincident with the experimental observations shown in Figs.2(d,e,f). The physical mechanism of the transition between the phase-locked behavior observed for short filaments [Fig.2(d)] and the phase dynamics observed for longer filaments [Fig.2(f)] originates from the combined effects of continuous dephasing between FW and SH waves inside the filament zone and velocity mismatch between the two-color pump and the THz pulses. The dephasing affects the electric field strength and the polarity of the generated terahertz waves while the velocity mismatch deteriorates the synchronization in the wavefront of $\mathrm{THz}$ radiation from each dipole distributed along the filament. These two effects are negligible for a short plasma channel, resulting in phase-locked behavior [10, 13]. In the case of a long filament, their combined work contributes to the dynamic phase evolution of the $\mathrm{THz}$ waves. For a quantitative comparison, we focus on the $\mathrm{THz}$ signals generated in four typical situations which are marked by dashed lines $I-I V$ in Figs.2(d,f,g,i). For a short plasma channel [3mm-long plasma, Fig.2(d,g)], the emitted THz electric field changes from maximum (I) to almost zero (II) when $\Delta \phi_{i}$ is shifted by $\pi / 2$. The simulated waveforms [Fig.2(1)] fit the measured ones [Fig.2(j)] very well. When the plasma extends to a 30-mm-long filament [Fig.2(f,i)], modification by $\pi / 2$ in $\Delta \phi_{i}$ enables variations in both the amplitude and the CEP of the emitted THz pulses. The calculated THz fields [Fig.2(m)] well reproduce the experimental observations [Fig.2(k)]. This good consistence between our LDA model and experiment indicates that it is essential to include the propagation effects both for the lasers and the $\mathrm{THz}$ waves in order to model the whole $\mathrm{THz}$ radiation properly.

To be compatible with detection performed by electrooptical sampling via ZnTe crystals, we limit our experimental investigation and the numerical evaluation of our model to low $\mathrm{THz}$ frequencies $(<4 T H z)$. However, our model suggests that this dynamic phase change with the filament length is a general phenomenon even if a much broader $\mathrm{THz}$ spectrum is sampled by using other detectors in experiment [29].

Using LDA model, we can calculate the angular distribution of the $\mathrm{THz}$ electric fields from a two-color filament. Based on the model, the THz emission is cylindrically symmetric to the axis of laser propagation. So we only consider the $\mathrm{THz}$ spatial distribution in the r-z plane [defined in Fig.1(a)]. As the first-born $\mathrm{THz}$ wave propagates to the position of $z=105 \mathrm{~mm}$, the calculated THz spatial distributions for $\Delta \phi_{i}=-\pi / 2$ are shown in Fig.3(a) for a 3-mm-long plasma and Fig.3(b) for a 30-mm-long filament. Each point in the figures represents integrated $\mathrm{THz}$ electric fields emitted from all dipoles located along the filament. Figures 3(a,b) suggest that, after $\mathrm{THz}$ radiation from a two-color filament propagating in air for a certain distance $(\sim 105 \mathrm{~mm})$, the coherent superposition of the nearly single-cycle THz electric fields emitted from all dipoles located along the filament results in an obvious enhancement in $\mathrm{THz}$ signal in a forward conical angle while fad- 
ing out in other directions. The conical angle for $\mathrm{THz}$ pulses emitted from a long filament [Fig.3(d)] is much sharper than that from a short plasma channel [Fig.3(c)]. In other words, a more directional $\mathrm{THz}$ source can be achieved by introducing a longer two-color filament, which is a result of the spatial coherence. The same behavior is observed in conical divergence of $\mathrm{THz}$ radiation from a one-color filament [7].

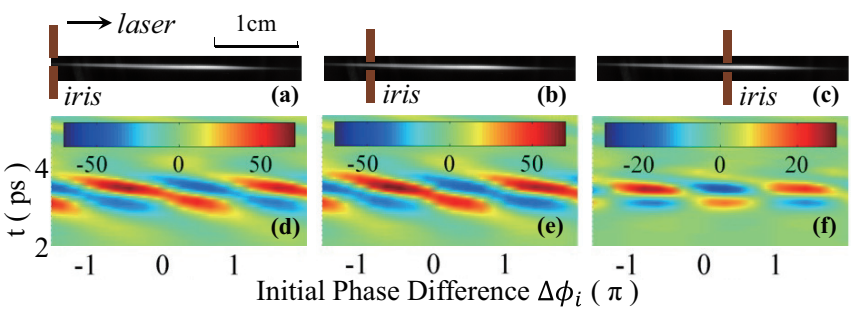

FIG. 4. The location of an iris is (a) $0 \mathrm{~mm}$, (b) $5 \mathrm{~mm}$, (c) $15 \mathrm{~mm}$ right to the beginning of the filament. (d)(e)(f) $\mathrm{THz}$ waveforms change with $\Delta \phi_{i}$ measured with an iris at the location of (a), (b) and (c), respectively.

In experiment, we verify the conical angle for $\mathrm{THz}$ emission from a 30-mm-long two-color filament by scanning a 2-mm-aperture metal iris along the filament, as shown in Figs.4(a,b,c). This aperture is large enough to survive the twocolor filament. When the iris is placed at the beginning of the filament, it does not have any effect on the forward $\mathrm{THz}$ radiation. Therefore, the measured $\mathrm{THz}$ signals [Fig.4(d)] are exactly the same as that obtained without an iris [Fig.2(f)]. When the iris is set $5 \mathrm{~mm}$ right to the beginning of the filament, the "effective" collection angle defined by this iris is around 11 degrees for $\mathrm{THz}$ pulses emitted from the first dipole located at the beginning of the filament and larger than 11 degrees for $\mathrm{THz}$ emission from other dipoles. In this situation, the measured $\mathrm{THz}$ signal [Fig.4(e)] is also quite similar to that observed without an iris. This means that the $\mathrm{THz}$ radiation from a 30-mm-long filament is confined in an emission angle less than 11 degrees. When the iris moves to the middle of the filament, i.e., the "effective" collection angle for $\mathrm{THz}$ radiation from the beginning dipole of the filament is about 4 degrees, the $\mathrm{THz}$ radiation from the whole filament is partially blocked by this iris. Consequently, the obtained $\mathrm{THz}$ amplitudes decrease a lot [see Fig.4(f)]. Meanwhile, the evolution of the THz pulses versus $\Delta \phi_{i}$ changes to a pattern when a filament is shorter. These observations show good agreement with our simulated result illustrated in Fig.3(d).

The evolution of the $\mathrm{THz}$ amplitudes versus the filament length is calculated by our LDA model, as shown in Fig.5. When the filament length is short ( $3 \mathrm{~mm}$ or less), the THz radiation increases rapidly with the filament length in the case of $\Delta \phi_{i}=-\pi / 2$. In contrast, the THz yield remains at 0 in the case of $\Delta \phi_{i}=0$. When the filament length is longer than 3 $\mathrm{mm}$, however, our simulation shows that $\mathrm{THz}$ radiation rises quickly with $\Delta \phi_{i}=0$. And it catches up with $\mathrm{THz}$ yield with $\Delta \phi_{i}=-\pi / 2$ at filament length of $15 \mathrm{~mm}$. For even longer filament length, the solid line (obtained with $\Delta \phi_{i}=-\pi / 2$ ) and

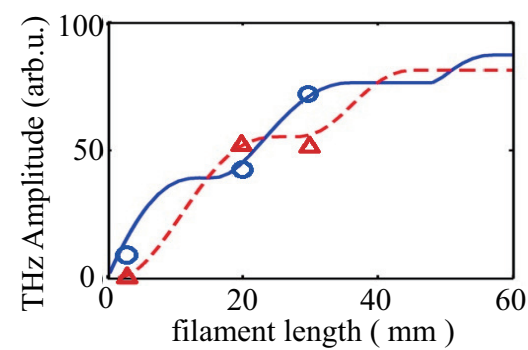

FIG. 5. THz amplitudes change with the filament length. The triangles show the experimental data obtained with $\Delta \phi_{i}=0$, and the dashed line is corresponding simulation result. The circles show the experimental data with $\Delta \phi_{i}=-\pi / 2$, and the solid line is corresponding simulation result.

the dashed line (obtained with $\Delta \phi_{i}=0$ ) intersect with each other several times, showing a common tendency of uprise with the increase of filament length. Both of these two simulated curves show good coincidence with the measured $\mathrm{THz}$ signals at the filament length of $3 \mathrm{~mm}, 20 \mathrm{~mm}$ and $30 \mathrm{~mm}$ in our experiment.

In summary, it has been shown that the spatio-temporal structure of a $\mathrm{THz}$ pulse emitted from a two-color laser filament can be controlled by manipulating the filament length and the initial phase difference between the two-color laser components. Besides, the $\mathrm{THz}$ radiation is confined in a cone and its angular distribution is dominated by the filament length. Furthermore, the peak intensity of the $\mathrm{THz}$ radiation emitted from a filament can be enhanced as the filament becomes longer with an increased pump energy. A LDA model can well describe the experimental observations, where the total $\mathrm{THz}$ radiation from a two-color laser filament is considered as a coherent superposition of $\mathrm{THz}$ waves emitted from individual dipoles distributed along the filament, and the $\mathrm{THz}$ wave propagation along the filament is taken into account. Such practical $\mathrm{THz}$ control techniques pave the way for promising $\mathrm{THz}$ applications.

We appreciate useful comments on this work from Dr. Feiyu Li. This work was supported by the National Basic Research Program of China (No. 2014CB339801) and the National Natural Science Foundation of China (Nos. 11474202 and 11421064). M. C. acknowledges support from the National 1000 Young Talent Program. Z. S. acknowledges the support of a Leverhulme Trust Research Grant and U.K. EPSRC (Grant No. EP/N028694/1).

* yanping.chen@sjtu.edu.cn

[1] B. Ferguson and X.-C. Zhang, Nat. Mater. 1, 26 (2002).

[2] L. Ho, M. Pepper, and P. Taday, Nat. Photon. 2, 541 (2008).

[3] G. Q. Liao, Y. T. Li, C. Li, L. N. Su, Y. Zheng, M. Liu, W. M. Wang, Z. D. Hu, W. C. Yan, J. Dunn, J. Nilsen, J. Hunter, Y. Liu, X. Wang, L. M. Chen, J. L. Ma, X. Lu, Z. Jin, R. Kodama, Z. M. Sheng, and J. Zhang, Phys. Rev. Lett. 114, 255001 (2015). 
[4] P. H. Siegel, IEEE Transactions on Microwave Theory and Techniques 50, 910 (2002).

[5] F. Theberge, M. Chateauneuf, J. Dubois, S. Desilets, and L.-S. Lussier, Opt. Express 17, 10841 (2009).

[6] H. Roskos, M. Thomson, M. Kreß, and T. Löffler, Laser Photon. Rev. 1, 349 (2007).

[7] C. D'Amico, A. Houard, M. Franco, B. Prade, A. Mysyrowicz, A. Couairon, and V. T. Tikhonchuk, Phys. Rev. Lett. 98, 235002 (2007).

[8] Y. Chen, T.-J. Wang, C. Marceau, F. Théberge, M. Châteauneuf, J. Dubois, O. Kosareva, and S. L. Chin, Appl. Phys. Lett. 95, 101101 (2009).

[9] A. Houard, Y. Liu, B. Prade, V. T. Tikhonchuk, and A. Mysyrowicz, Phys. Rev. Lett. 100, 255006 (2008).

[10] M. Kress, T. Löffler, S. Eden, M. Thomson, and H. G. Roskos, Opt. Lett. 29, 1120 (2004).

[11] D. J. Cook and R. M. Hochstrasser, Opt. Lett. 25, 1210 (2000).

[12] X. Xie, J. Dai, and X.-C. Zhang, Phys. Rev. Lett. 96, 075005 (2006).

[13] K. Y. Kim, J. H. Glownia, A. J. Taylor, and G. Rodriguez, Opt. Express 15, 4577 (2007).

[14] V. B. Gildenburg and N. V. Vvedenskii, Phys. Rev. Lett. 98, 245002 (2007).

[15] M. Chen, A. Pukhov, X.-Y. Peng, and O. Willi, Phys. Rev. E 78, 046406 (2008).

[16] H.-C. Wu, J. Meyer-ter-Vehn, and Z.-M. Sheng, New J. Phys. 10, 043001 (2008).

[17] W.-M. Wang, Z.-M. Sheng, H.-C. Wu, M. Chen, C. Li, J. Zhang, and K. Mima, Opt. Express 16, 16999 (2008).

[18] I. Babushkin, W. Kuehn, C. Köhler, S. Skupin, L. Bergé, K. Reimann, M. Woerner, J. Herrmann, and T. Elsaesser, Phys.
Rev. Lett. 105, 053903 (2010).

[19] V. A. Andreeva, O. G. Kosareva, N. A. Panov, D. E. Shipilo, P. M. Solyankin, M. N. Esaulkov, P. González de Alaiza Martínez, A. P. Shkurinov, V. A. Makarov, L. Bergé, and S. L. Chin, Phys. Rev. Lett. 116, 063902 (2016).

[20] M. Clerici, M. Peccianti, B. E. Schmidt, L. Caspani, M. Shalaby, M. Giguère, A. Lotti, A. Couairon, F. Légaré, T. Ozaki, D. Faccio, and R. Morandotti, Phys. Rev. Lett. 110, 253901 (2013).

[21] V. A. Kostin, I. D. Laryushin, A. A. Silaev, and N. V. Vvedenskii, Phys. Rev. Lett. 117, 035003 (2016).

[22] Y. Shen, T. Watanabe, D. A. Arena, C.-C. Kao, J. B. Murphy, T. Y. Tsang, X. J. Wang, and G. L. Carr, Phys. Rev. Lett. 99, 043901 (2007).

[23] D. Turchinovich, J. M. Hvam, and M. C. Hoffmann, Phys. Rev. B 85, 201304 (2012).

[24] A. Leitenstorfer, K. A. Nelson, K. Reimann, and K. Tanaka, New J. Phys. 16, 045016 (2014).

[25] A. Couairon and A. Mysyrowicz, Phys. Rep. 441, 47 (2007).

[26] S. L. Chin, S. A. Hosseini, W. Liu, Q. Luo, F. Théberge, N. Aközbek, A. Becker, V. P. Kandidov, O. G. Kosareva, and H. Schroeder, Can. J. Phys. 83, 863 (2005).

[27] B. M. Penetrante and J. N. Bardsley, Phys. Rev. A 43, 3100 (1991).

[28] D. J. Griffiths and R. College, Introduction to electrodynamics, Vol. 3 (prentice Hall Upper Saddle River, NJ, 1999).

[29] N. Karpowicz, J. Dai, X. Lu, Y. Chen, M. Yamaguchi, H. Zhao, X.-C. Zhang, L. Zhang, C. Zhang, M. Price-Gallagher, C. Fletcher, O. Mamer, A. Lesimple, and K. Johnson, Appl. Phys. Lett. 92, 011131 (2008). 